and social functions. The aim of this study was to assess the impact of disease activity on HRQoL.

Methods This was a cross-sectional descriptive study conducted at Kenyatta National Hospital rheumatology and renal outpatient clinics. 62 patients fulfilling $\geq 4$ Systemic Lupus International Collaborating Clinics Criteria (SLICC) 2012 for classification of SLE were consecutively recruited. 27 patients with overlap syndromes were excluded. Disease activity was assessed by the modified Systemic Lupus Erythematosus Disease Activity Index 2000 (cSLEDAI-2K). HRQoL was evaluated using self-administered LupusQoL with scores ranging from 0 (worst) to 100 (best). HRQoL was correlated with age, disease duration and disease activity. Data analysis was performed on SPSS version 23.

Results The study comprised 60 female patients with mean age $34.7 \pm 11.8$ years. The median disease duration was 36 months and ranged from 1-324 months. Mean cSLEDAI score was $7 \pm 5.2$ and median disease activity score was 7 . Renal involvement occurred in $53.2 \%$.

All domains of LupusQoL were impaired. The mean LupusQoL score was 56\% 24.4 (figure 1). SLEDAI scores inversely correlated with scores of physical health, pain, burden to others, body image and general health. The patients with renal disease had significantly lower QoL compared to other patients. Age and disease duration were positively correlated with QoL. Disease duration was associated with a better QoL in the pain, emotional health and body image domains.

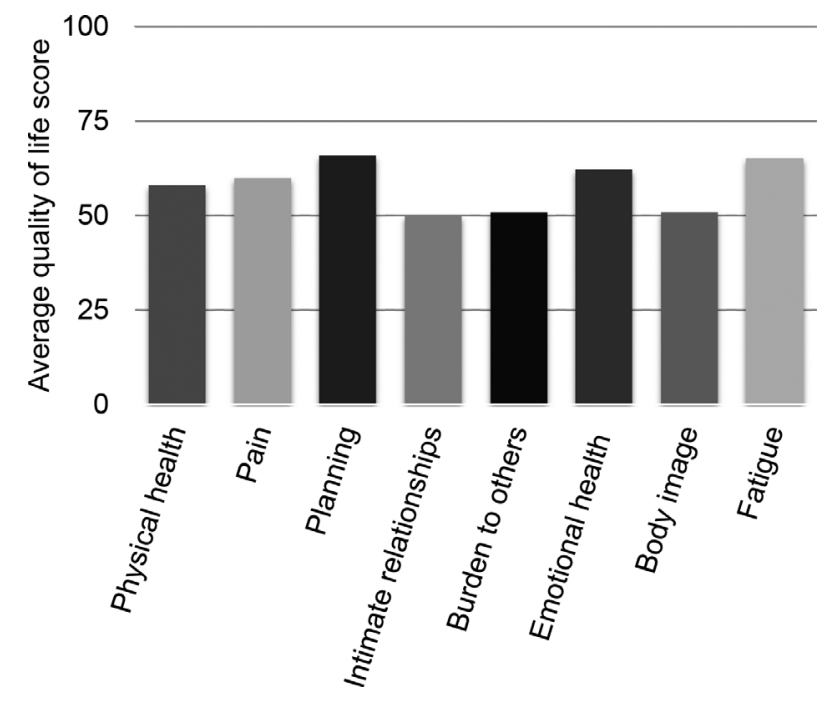

Abstract P188 Figure 1 Quality of life domains

Conclusions Our study showed a low HRQoL in those with active disease. Young age, a recent diagnosis of lupus and presence of renal disease was associated with a poorer QoL.

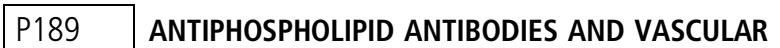 RENAL LESIONS AS PROGNOSTIC FACTORS IN LUPUS NEPHRITIS}

\footnotetext{
${ }^{1}$ Annamaria Paglionico, ${ }^{1}$ Valentina Varriano, ${ }^{2}$ Luca Petricca, ${ }^{1}$ Clara Di Marioa, ${ }^{2}$ Maria Rita Gigante, 'Giacomo Tanti, 'Barbara Tolusso, 'Gianfranco Ferraccioli, 1,2Elisa Gremese. ${ }^{1}$ Division of Rheumatology, Università Cattolica del Sacro Cuore, Rome; ${ }^{2}$ Division of Rheumatology, Fondazione Policlinico Universitario 'A. Gemelli'- I.R.C.C.S., Rome, Italy
}

10.1136/lupus-2020-eurolupus.231
Purpose To determine the role of antiphospholipid antibodies (aPL) and vascular renal lesions on renal prognosis, in terms of time to achieve remission, number of renal flares and development of chronic renal damage in patients with lupus nephritis (LN).

Methods 91 consecutive LN patients have been evaluated and the follow-up data have been collected at the baseline and at $6,12,24$ months and at the last follow-up visit. Histopathological data of 41 patients were evaluated according to the 2016 revision of ISN/RPS classification.

Results Among the $91 \mathrm{LN}$ patients, 31(34.1\%) were aPL positive (aPL +$), 10(32.2 \%)$ of them were affected by Antiphospholipid Antibodies Syndrome (APS), 53.3\% showed a single aPL positivity, $23.1 \%$ double aPL positivity and $15.4 \%$ triple aPL positivity. At the last follow up visit a significant higher number of aPL+ patients showed a persistent complement consumption than aPL negative (aPL-) patients $(p=0.001)$. We observed that aPL- patients showed a remission achievement time slightly earlier than aPL+ patients $(13.6 \pm 1.0$ months vs $16.5 \pm 1.5$ months; log-rank test: $p=0.06$, Breslow test: $\mathrm{p}=0.08$ ) and as expected, patients with a persistent complement consumption achieve remission later (18.2 \pm 1.5 months vs $13.0 \pm 1$ months; log-rank test: $p=0.002$, Breslow test: $\mathrm{p}=0.003)$. Furthermore at the last follow up, a significant higher percentage of aPL+ patients developed persistent proteinuria $(p=0.02)$ and chronic renal failure $(p=0.04)$. Considering histolopathologic features we didn't observe significant differences between aPL+ and aPL- patients but we found two typical vascular lesions (mesangiolysis and vascular thrombi) only in aPL+ patients.

Conclusion Apl positivity is a predictor of worse renal outcome but in our cohort we didn't find an association between aPL positivity and vascular renal lesions at renal biopsy. The worse renal outcome and the late time to achieve remission in aPL+ group can be related to a cumulative vascular damage over time.

\section{P190 A SIMPLIFIED APPROACH FOR PATIENTS WITH SLE TO REPORT DISEASE ACTIVITY USING A REVISED VERSION OF THE SWEDISH SYSTEMIC LUPUS ACTIVITY QUESTIONNAIRE}

${ }^{1,2}$ Susanne Pettersson, ${ }^{1}$ Vera Illescas-Bäckelin, ${ }^{3}$ Andreas Jönsen, ${ }^{1}$ Iva Gunnarsson,
${ }^{4}$ Estelle Trysberg, ${ }^{5}$ Dag Leonard, ${ }^{6}$ Christopher Sjöwall, ${ }^{1}$ Elisabet Svenungsson.
${ }^{1}$ Rheumatology Unit, Dept. of Medicine, Solna, Karolinska Institutet, Karolinska University
Hospital, Stockholm; ${ }^{2}$ Dept. of Neurobiology, Care Sciences and Society, Karolinska
Institutet, Stockholm; ${ }^{3}$ Dept. of Clinical Sciences Lund, Rheumatology, Lund University,
Lund; ${ }^{4}$ Dept. of Rheumatology, Sahlgrenska University Hospital, Göteborg; ${ }^{5}$ Dept. of
Medical Sciences, Science for Life Laboratory, Rheumatology, Uppsala University, Uppsala;
${ }^{6}$ Rheumatology/Division of Neuro and Inflammation Sciences, Dept. of Clinical and
Experimental Medicine, Linköping University, Linköping, Sweden

10.1136/lupus-2020-eurolupus.232

Background/Purpose We compared patients' assessments of SLE disease activity, reported by the SWE-SLAQr, with physicians' assessments using SLE activity measure (SLAM) and SLE disease activity index (SLEDAI-2K).

Methods Patients ( $n=115)$, median age 43 (IQR 24) years, disease duration 15 (IQR 17) years filled out SWE-SLAQr prior to physicians' assessments. Correlations (Spearman's $\rho$ ) were calculated between SWE-SLAQr-total, sub-scales (Symptom score, Patients global) and physicians SLAM, SLEDAI-2K with and excluding the laboratory items, further corresponding items in SLAQ and SLAM were explored. 
Results Correlations between patients' and physicians' assessments were higher for SLAM-nolab: SWE-SLAQr total, $\rho=0.69$, Symptom score, $\rho=0.67$, and Patients global, $\rho=0.68$ than for SLAM: SWE-SLAQr total, $\rho=0.51$, Symptom score, $\rho=0.49$, and Patients global, $\rho=0.53$. The items fatigue $(\rho=0.72)$ and alopecia $(\rho=0.71)$ showed highest degree of correlation, and dyspnea/ pleuritic chest pain had the lowest correlation between patients' and physicians' assessments $(\rho=0.19, \mathrm{p}=0.039)$. Correlations with SLEDAI-nolab were lower $(\rho \leq 0.36)$ for all subscales. No correlations were found between patients' and physicians' assessments when using SLEDAI-2K $(\rho<0.09$ for all).

Conclusions We conclude that SWE-SLAQr performed equally well as SLAQ, demonstrating that the shorter version can be used to monitor disease impact. We encourage further use of SWE-SLAQr and recommend its implementation in clinical care, we believe it is especially well suited to support digital and telephone contacts. However further attention is needed to evaluate the discrepancy between physicians' and patients' evaluation of thoracic pain/symptoms.

\section{P191 CHINESE SYSTEMIC LUPUS ERYTHEMATOSUS TREATMENT AND RESEARCH GROUP (CSTAR) REGISTRY - SUBJECTIVE WELL-BEING IN PATIENTS WITH SYSTEMIC LUPUS ERYTHEMATOSUS}

\begin{abstract}
${ }^{1}$ Yue Shi, ${ }^{2,3}$ Dandan Bi, ${ }^{4}$ Yanhong Wang, ${ }^{5}$ Ruofan Li, ${ }^{6}$ Lijun Wu, ${ }^{7}$ Cheng Zhao, ${ }^{8}$ Zhenbiao $\mathrm{Wu},{ }^{9}$ Xinwang Duan, ${ }^{10}$ Jian $\mathrm{Xu},{ }^{11}$ Feng Zhan, ${ }^{12}$ Min Yang, ${ }^{13}$ Shengyun Liu, ${ }^{14}$ Qin Li, 'Shuo Zhang, 'Lingshan Liu, 'Jiuliang Zhao, 'Xinping Tian, ${ }^{2,3}$ Xinying Li, ${ }^{1}$ Qian Wang, 'Xiaofeng Zeng, CSTAR Co-authors. 'Dept. of Rheumatology, Peking Union Medical College Hospital, Peking Union Medical College and Chinese Academy of Medical Sciences, National Clinical Research Center for Immunologic Diseases, Ministry of Science and Technology, Key Laboratory of Rheumatology and Clinical Immunology, Ministry of Education, Beijing; ${ }^{2}$ CAS Key Laboratory of Mental Health, Institute of Psychology, Beijing; ${ }^{3}$ University of Chinese Academy of Sciences, Beijing; ${ }^{4}$ Dept. of Epidemiology and Biostatistics, Institute of Basic Medical Sciences Chinese Academy of Medical Sciences, School of Basic Medicine Peking Union Medical College, Beijing; ${ }^{5}$ Dept. of International Education, The Experimental High School Attached to Beijing Normal University, Beijing; ${ }^{6}$ Dept. of Rheumatology, The People's Hospital of Xinjiang Autonomous, Urumqi; ${ }^{7}$ Dept. of Rheumatology, First Affiliated Hospital of Guangxi Medical University, Nanning; ${ }^{8}$ Dept. of Clinical Immunology and Rheumatology, Xijing Hospital Affiliated with The Fourth Military Medical University, Xi'An; ${ }^{9}$ Dept. of Rheumatology, The Second Affiliated Hospital of Nanchang University, Nanchang; ${ }^{10}$ Dept. of Rheumatology, First Affiliated Hospital of Kunming Medical University, Kunming; ${ }^{11}$ Dept. of Rheumatology, Hainan Provincial People's Hospital, Haikou; ${ }^{12}$ Dept. of Rheumatology, Nanfang Hospital, Southern Medical University, Guangzhou; ${ }^{13}$ Dept. of Rheumatology, First Affiliated Hospital of Zhengzhou University, Zhengzhou; ${ }^{14}$ Dept. of Rheumatology, The First People's Hospital of Yunnan Province, Kunming, China
\end{abstract}

10.1136/lupus-2020-eurolupus.233

Objectives Systemic lupus erythematosus (SLE) can significantly influence patients' quality of life and subjective wellbeing (SWB). This study compared SWB statuses in SLE patients and analysed their relationship with clinical manifestations, emotional variables and related positive factors.

Methods Overall, 1779 SLE patients from the Chinese SLE Treatment and Research Group (CSTAR) and 203 age and gender-matched individuals from the general population without self-reported SLE were invited to complete measures of SWB, emotional variables and related positive factors. These patients' clinical data in CSTAR were also linked to investigate the influence of clinical manifestations and psychological factors on SWB among SLE patients.

Results SWB, self-efficacy and resilience were significantly lower $(\mathrm{P}<0.001)$ in SLE patients than in the general population, and self-esteem, depression and anxiety were significantly higher $(\mathrm{P}<0.001)$. Among the SLE patients, life satisfaction was significantly positively correlated with patients' age $(\mathrm{P}<0.001)$ and the age at diagnosis $(\mathrm{P}<0.001)$ and negatively correlated with SLE disease activity index (SLEDAI) $(\mathrm{P}<0.05)$. Premature gonadal failure $(\mathrm{PGF}) \quad(\mathrm{P}<0.05)$ was significantly associated with positive and negative affect scores. In active SLE patients with $\operatorname{SLEDAI} \geq 4$, depression and resilience were life satisfaction predictors. However, anxiety, selfesteem and resilience predicted life satisfaction changes in in patients with SLEDAI $<4$.

Conclusion SWB provides useful insight into the impact of SLE on mental health and opportunities to improve quality of life and clinical care. PGF and disease activity emerge as are predictors of SWB status and potential therapeutic targets to develop positive attitude in SLE patients.

\section{P192 EVALUATION OF PREDICTIVE FACTORS OF WORSE PROGNOSIS IN LUPUS NEPHRITIS: FOCUS ON NEW PATHOGENETIC PATHWAYS}

${ }^{1}$ Valentina Varriano, ${ }^{1}$ Annamaria Paglionico, ${ }^{2}$ Luca Petricca, ${ }^{1}$ Clara Di Mario, ${ }^{2}$ Maria Rita Gigante, ${ }^{1}$ Giacomo Tanti, ${ }^{2}$ Barbara Tolusso, 1,2Elisa Gremese. 'Institute of Rheumatology, Università Cattolica del Sacro Cuore, Rome, Italy; ${ }^{2}$ Division of Rheumatology, Fondazione Policlinico Universitario 'A. Gemelli'- I.R.C.C.S., Rome

\subsection{6/lupus-2020-eurolupus.234}

Purpose To evaluate the prognostic factors in a cohort of patients with lupus nephritis (LN) focusing on of the IL-17, 23 axis as new pathogenetic pathway.

Patients and Methods $91 \mathrm{LN}$ patients were enrolled. Laboratory, immunological and disease activity data were collected at the baseline and at 6(T6),12(T12),24(T24) months and at the last follow-up(FU).84 renal biopsies were evaluated according to ISN/RPS classification, assessing the inflammatory interstitial infiltrate using the BANFF score. Baseline serum levels of IL17 and IL-23 were assessed by ELISA in 37 patients.

Results Among the 84 renal biopsies evaluated $77 \%$ belonged to class III and IV; $41,8 \%$ of patients had an interstitial infiltrate $<5 \%, 35.2 \%$ between $5 \%$ and $25 \%$ and $15,4 \%$ above $25 \%$. Regarding immunological data $35,2 \%$ of patients revealed a seropositivity for antiphospholipid antibodies (APL $+)$. Serum level of IL-17 and IL-23 were $0.12 \pm 0.15 \mathrm{pg} / \mathrm{ml}$ and $27.7 \pm 9.12 \mathrm{pg} / \mathrm{ml}$ respectively. Through the ROC curves analysis we found a cut off value of $25.89 \mathrm{pg} / \mathrm{ml}$ of IL-23 for remission at T6. Among the 10 patients with a IL-23 level above this cut-off none achieved remission at T6 and at the univariate analysis a serum level of IL-23 above the cut-off was associated with an interstitial infiltrate $>5 \%$ at renal biopsy and persistent proteinuria. Finally, we conducted an univariate and multivariate analysis for each renal outcome considered. We found that an inflammatory interstitial infiltrate $>5 \%$ and APL+ were associated with worse renal outcome in terms of early and persistent remission, chronic damage, persistent proteinuria, and renal flare both in univariate and multivariate analysis. Higher serum level of IL-23 was associated with persistent proteinuria, renal flare and tended to be associated to chronic renal damage.

Conclusion Interstitial infiltrate and APL + resulted as the strongest predictors of worse renal outcome. An higher serum level of IL-23 resulted as a negative prognostic factor highlighting its possible role as a biomarkers of more aggressive disease. 